\title{
Interference between object-based attention and object-based memory
}

\author{
Michi Matsukura and Shaun P. Vecera \\ University of Iowa, Iowa City, Iowa
}

\begin{abstract}
Research has shown that there are at least two kinds of visual selective attention: location based and object based. In the present study, we sought to determine the locus of spatially invariant object-based selection using a dual-task paradigm. In four experiments, observers performed an attention task (object feature report or visual search) with a concurrent memory task (object memory or spatial memory). Object memory was interfered with more by a concurrent object-based attention task than by a concurrent location-based attention task. However, this interference pattern was reversed for spatial memory, with greater interference by a location-based attention task than by an object-based attention task. These findings suggest that object-based attention and locationbased attention are functionally dissociable and that some forms of object-based selection operate within visual short-term memory.
\end{abstract}

Visual attention selects objects as well as locations; observers can identify two attributes of a single object more accurately than two attributes of two different objects. For example, when two superimposed objects were shown, observers were more accurate at reporting two target attributes from one object rather than from two different objects (Awh, Dhaliwal, Christensen, \& Matsukura, 2001; Duncan, 1984; Vecera \& Farah, 1994).

Despite numerous demonstrations of object-based effects of attention, the locus of object-based attention is less well established. Vecera and Farah (1994; Vecera, 1994; see also Awh et al., 2001) suggested that there were two forms of object-based selection. Objects could be selected from a relatively late selection stage that represents the shape of an object and combines that object's features. In such a representation, an object's shape (i.e., features and parts) is selected, not the object's location. Objects could also be selected from a relatively early grouped array representation, which allows locations to be selected by spatial attention. In this grouped array selection, object-based attention arises because locations within an object are perceptually grouped, whereas locations between objects are weakly grouped. Distinguishing these two forms of object-based selection has been difficult, and the literature has focused on a grouped array interpretation of object-based effects.

In the present experiments, we asked whether some object-based effects of attention can occur at a relatively late visual processing stage that operates after objects have disappeared and are no longer available to perceptual processes. One candidate for such a late object-based attention process is visual short-term memory (VSTM). VSTM holds a small number of items across an eye movement
(Hollingworth, Richard, \& Luck, 2008; Irwin, 1992; Irwin \& Andrews, 1996) or across a temporal delay without an eye movement (Luck \& Vogel, 1997; Vogel, Woodman, \& Luck, 2001). VSTM can maintain approximately three to four objects, irrespective of the number of features that an object possesses (e.g., Awh, Barton, \& Vogel, 2007; Gajewski \& Brockmole, 2006; Luck \& Vogel, 1997; Vogel et al., 2001; but see Alvarez \& Cavanagh, 2004).

Previous research on the relationship between visual memory and attention has focused primarily on spatial memory and spatial attention. For example, spatial attention acts as a rehearsal mechanism for spatial memory. Observers are faster to perform perceptual discriminations on targets appearing at locations held in spatial memory than on targets appearing elsewhere (Awh, Jonides, \& ReuterLorenz, 1998). Similarly, the rate of spatial visual search slows down when observers perform a concurrent spatial memory task (Oh \& Kim, 2004; Woodman \& Luck, 2004; but see Woodman, Vogel, \& Luck, 2001).

Some research has investigated the relationship between object memory and object-based attention. Barnes, Nelson, and Reuter-Lorenz (2001) asked observers to maintain an object in visual memory while performing an object-based attention task that required them to decide which of two vertices of a hexagonal object was located higher than the other (a vertex judgment task). Observers' responses were faster when these vertices were part of a single object than when they were part of two different objects (Baylis \& Driver, 1993). Interestingly, when a complex object was concurrently maintained in observers' VSTM, the size of this object-based effect was reduced (Barnes et al., 2001). Specifically, there was a smaller advantage for attending to a single object than to two ob-

M. Matsukura, michi-matsukura@uiowa.edu 
jects when a complex object was stored in visual memory than when no object was stored in visual memory. Thus, Barnes et al.'s data suggest that object-based attention and object memory involve similar processes.

Despite the importance of the foregoing results, previous research using the vertex judgment task suggests that this task might have not tapped into late object-based attention mechanisms. Gibson (1994) demonstrated that the object-based effect observed in the vertex judgment task actually might reflect observers' sensitivity to lower level perceptual cues - namely, convexity. When the two vertices were part of a single hexagon, this hexagon presented itself as a convex object. Gibson found that observers' vertex judgments were faster for convex objects than for concave objects, irrespective of the number of objects. Moreover, it is highly likely that the vertex judgment task did not require observers to rely on VSTM, because the stimuli remained on the display until observers' response. These considerations suggest that a concurrent memory task might have affected observers' sensitivity to perceptual cues or interpretations of figure-ground relations, instead of influencing object-based attention.

In the present experiments, we examined whether an object-based attention task could interfere with observers' performance of a concurrent object memory task but not of a concurrent spatial memory task. If some object-based attentional effects arise from objects stored in VSTM, then an object-based attention task should interfere with an object memory task, but not with a spatial memory task. Conversely, a spatial attention task should interfere with a spatial memory task, but not with an object memory task. We tested these predictions by crossing two attention tasks (object feature report and spatial visual search) with two memory tasks (object memory and spatial memory). Four groups of observers participated in one of the four experimental pairings (object-attention/object-memory, object-attention/spatial-memory, spatial-attention/objectmemory, and spatial-attention/spatial-memory).

Each group of observers experienced three conditions: memory only, attention only, and memory and attention. In the memory-only condition, observers remembered either the colors of two objects (object memory) or the locations of two objects (spatial memory). In the attentiononly condition, observers reported features from one or two objects (object feature report) or searched for a target among an array of distractors (spatial visual search). In the memory-and-attention condition, within a single trial, observers performed either the object memory or the spatial memory task and either the object feature report or the visual search task. The sequence of events was identical across all conditions (see Figure 1).

In our experiments, an attention task was performed during a memory retention interval (i.e., after the offset of to-be-remembered objects/locations and before observers' report of identity/location of the object). Pilot results indicated that the dual-task performance influenced observers' memory task performance more than it affected attention task performance. Accordingly, we focused on the accuracy decrements in memory task performance when an attention task was concurrently performed. We
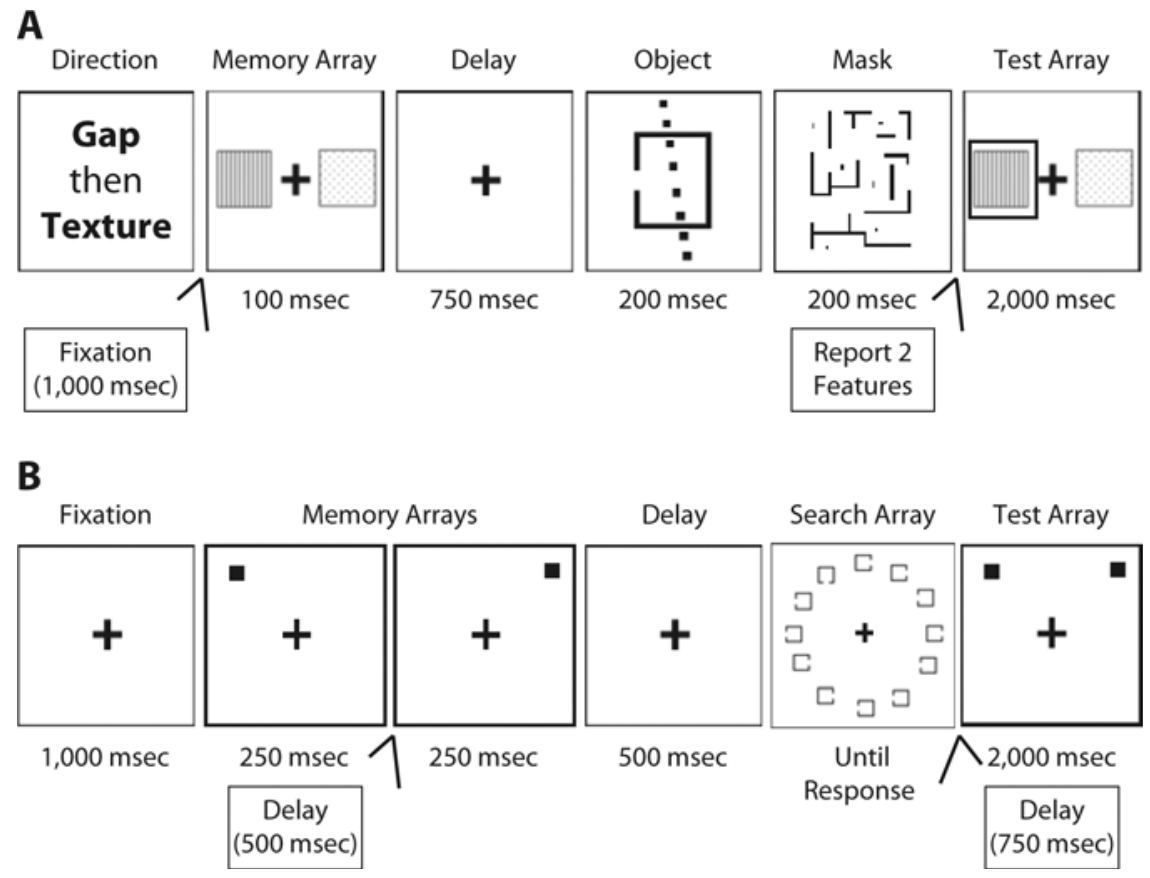

Figure 1. (A) Event sequence for a memory-and-attention trial in the object-attention/ object-memory experiment. (B) Event sequence for a memory-and-attention trial in the spatial-attention/spatial-memory experiment. Stimuli are not to scale. Different fill patterns are used to represent different colors. 
predicted that object memory would be impaired more by a concurrent object-based attention task than by a concurrent spatial attention task, whereas spatial memory would be impaired more by a concurrent spatial attention task than by a concurrent object-based attention task.

\section{Participants}

\section{METHOD}

For each of the four experiments, observers were 48 University of Iowa undergraduates who received partial course credit for their involvement; all reported having normal or corrected-to-normal vision. ${ }^{1}$

\section{Stimuli}

Stimuli were viewed from a distance of $60 \mathrm{~cm}$ and were presented on a black background with a continuously visible fixation cross, except when target objects and masks for the object feature report task were presented.

The object feature report task. Stimuli consisted of two superimposed objects: a box and a line (Figure 1A). Each object had two dimensions. The box was either short or tall and had a gap on either the left or the right. The line was either dotted or dashed and tilted to either the left or the right. The width of each box was $0.67^{\circ}$ of visual angle. The box was either $1.14^{\circ}$ or $0.86^{\circ}$ in height. The box's gap $\left(0.20^{\circ}\right.$ wide $)$ was centered on either the left or the right of the box. The line was $1.53^{\circ}$ long and tilted $8^{\circ}$ clockwise (rightward) or counterclockwise (leftward) from the vertical position. The line was either dotted or dashed; both a dot and a dash contained four pixels, but the configuration differed. The pattern mask was $2.1^{\circ}$ in width and $1.62^{\circ}$ in height.

The object memory task. Two colored squares $\left(1.1^{\circ} \times 1.1^{\circ}\right)$ were presented $1.9^{\circ}$ to the left and right of fixation. The squares' colors were selected randomly (without replacement) from a set of seven easily discriminable colors: violet, red, blue, green, yellow, gray, and brown.

The visual search task. The search arrays consisted of 4 or 12 Landolt $C$ s that measured $0.45^{\circ} \times 0.45^{\circ}\left(0.08^{\circ}\right.$ line thickness $)$, with a $0.12^{\circ}$ gap on the left or the right side. The target was an identical square with a gap on either the top or the bottom. For a set size of 12 , the stimuli were presented at 12 locations evenly spaced on an imaginary circle, with a radius of $3.8^{\circ}$, which was centered at fixation. To maintain the same display density across set sizes, for a set size of 4 , the stimuli were presented at 4 locations on a randomly chosen quarter of the imaginary circle.

The spatial memory task. Two white dots $\left(0.13^{\circ} \times 0.13^{\circ}\right)$ indicated to-be-remembered locations. Each dot appeared at a randomly selected location within a $3.3^{\circ} \times 3.3^{\circ}$ region centered in the middle of the display. However, these dots did not appear within a $1.9^{\circ} \times$ $1.9^{\circ}$ region centered at the fixation, which corresponded to the area in which the box-line stimuli and masks for the object feature report task appeared. We used this restriction to avoid masking between the stimuli. Each dot was centered at least $0.67^{\circ}$ from the center of the other dot's location.

\section{Procedure}

Each trial began with an articulatory suppression task, in which observers spoke either "A, B, C, D" or "1, 2, 3, 4" aloud throughout the trial. Observers were instructed to speak at a rate of three or four digits or letters per second, and the experimenter monitored observers to ensure adequate performance and to prevent verbal recoding of visual information (e.g., Besner, Davies, \& Daniels, 1981).

Observers performed three blocks in a single experimental session. After a few minutes of practice with memory and attention trials, each observer completed three experimental blocks (attention only, memory only, memory and attention) of 128 trials. Block order was counterbalanced across observers. For all four experiments, in the attention-only condition, the test array of the memory task disappeared 1,500 msec after its onset without the observer's response.
Similarly, in the memory-only condition, the attention task displays (i.e., two question displays for the object feature report task, a search array for the visual search task) disappeared $1,500 \mathrm{msec}$ after their onset. We chose a 1,500-msec duration because it was an average of how long observers took to perform each attention and memory task in the memory-and-attention condition.

Object-attention/object-memory experiment. Observers were told two to-be-reported features of objects at the beginning of each block. The memory array for the object memory task appeared for $100 \mathrm{msec}$ after a 1,000-msec fixation screen (Figure 1A). The offset of the memory array was followed by a blank delay period of $750 \mathrm{msec}$; then the box-line stimuli for the object feature report task appeared for $200 \mathrm{msec}$ and were immediately masked for $200 \mathrm{msec}$. Then, two question screens for two to-be-reported features appeared; as soon as observers identified the first feature, the second feature report question appeared. Observers made an unspeeded response to report the relevant features by pressing one of the two buttons. For example, observers pressed " 1 " if the gap was located on the left side of the box and " 2 " if the gap was located on the right side of the box. Observers reported all possible pairings of objects' features. The offset of the second question screen was followed by the test array of the object memory task; this test array appeared for 2,000 msec. Observers made an unspeeded response to indicate whether the probed item on the test array was identical with the item on the same location of the memory array. Observers pressed " 1 " if the two arrays were identical and " 2 " if these arrays were different by one item's color. The test array was identical to the memory array on $50 \%$ of the trials.

Spatial-attention/object-memory experiment. The event sequence of a spatial-attention/object-memory trial was identical to that of the object-attention/object-memory trial, with the exception of the search array. After a $750-\mathrm{msec}$ blank delay period following the offset of the memory array, either 4 or 12 search objects appeared and remained until observers responded. Observers made a speeded response to the search array, indicating whether the top gap or the bottom gap target was present; observers pressed " 1 " if the target was present and " 2 " if the target was absent. A target was present on $50 \%$ of the trials and absent for $50 \%$ of the trials. The offset of the search array was followed by another $750-\mathrm{msec}$ blank period and then by the test array. Observers performed the object memory task as in the object-attention/object-memory experiment.

Spatial-attention/spatial-memory experiment. While the visual search task used in the spatial-attention/object-memory experiment was kept constant, observers performed a concurrent spatial memory task. Each trial began with the sequential presentation of two white dots that were used to indicate two to-be-remembered locations (Figure 1B). After the presentation of a 1,000-msec fixation screen, each dot was presented for $250 \mathrm{msec}$, separated by a $500-\mathrm{msec}$ blank period. After the offset of the second dot, there was a delay of $750 \mathrm{msec}$, followed by the search array. Observers' response to the search task was followed by another 750-msec blank period and the test array, in which both dots were presented simultaneously for $2,000 \mathrm{msec}$. On $50 \%$ of the trials, two dots were shown at the same locations during the serial memory array presentations. On the other $50 \%$ of the trials, one of the dots was presented at a randomly chosen new location. Observers made an unspeeded response to indicate whether a location change was detected. The observers pressed " 1 " if the two dots' locations on the memory arrays were the same as those on the test array and "2" if one of those dots' locations was different.

Spatial-attention/object-memory experiment. Observers performed the object feature report task used in the object-attention/ object-memory experiment and the spatial memory task used in the spatial-attention/spatial-memory experiment.

\section{RESULTS}

Figure 2 depicts memory task accuracy when observers performed a memory task alone (memory-only con- 
dition) and with a concurrent attention task (memoryand-attention condition). In the spatial-attention/ object-memory and spatial-attention/spatial-memory experiments, to ensure that observers' attention was strongly engaged, we focused on memory accuracy for the set size 12 trials rather than on that for the set size 4 trials. We found the same pattern of results when memory accuracy for set size 12 was replaced with that for set size 4 ; for set size 4, the accuracy drop from the memory-only condition to the memory-and-attention condition was significant for both spatial-attention/object-memory and spatial-attention/spatial-memory experiments $(p \mathrm{~s}<$ $.0001)$. A significant two-factor ANOVA with betweensubjects factors of attention task type and memory task type indicated that performing concurrent attention tasks produced a larger decrement on memory performance in the object-attention/object-memory and spatial-attention/ spatial-memory conditions than in the other two conditions $[F(1,188)=8.4, p<.004]$.

\section{Memory and Attention Performance}

The accuracy drop from the memory-only condition to the memory-and-attention condition was significant for all four experiments $(p \mathrm{~s}<.0001)$. Most impor- tantly, as predicted, the size of interference was larger when an object attention task was performed with a concurrent object memory task than when it was performed with a spatial memory task. Furthermore, this interference pattern was reversed for the visual search task. The size of interference was smaller when a spatial attention task was performed with a concurrent object memory task than when it was performed with a spatial memory task. These observations were supported by a two-factor ANOVA with between-subjects factors of attention task type (object feature report vs. visual search) and memory task type (object memory vs. spatial memory). ${ }^{2}$ The observed interaction between the attention and memory tasks was significant $[F(1,188)=$ $35.4, p<.0001]$, indicating that performing concurrent attention tasks produced larger decrements on memory performance in the object-attention/object-memory and spatial-attention/spatial-memory conditions than in the other two conditions.

Neither the main effect of attention task nor the main effect of memory task was significant ( $p$ s $>$.2). Pairwise comparisons confirmed that the $15 \%$ interference effect observed in the object-attention/object-memory experiment was significantly larger than the $7 \%$ interference ef-
Object-Attention/Object-Memory

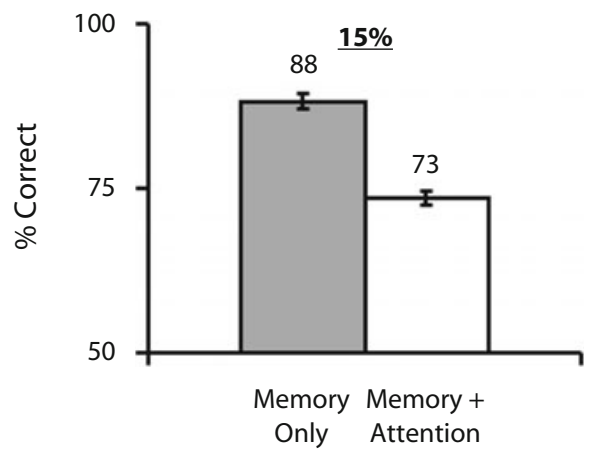

Spatial-Attention/Object-Memory

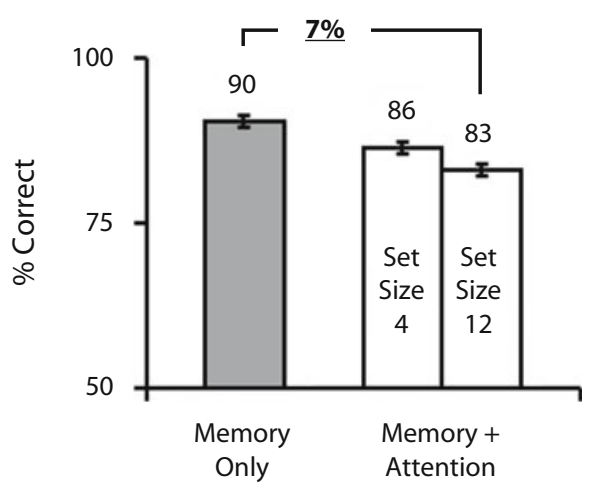

Object-Attention/Spatial-Memory

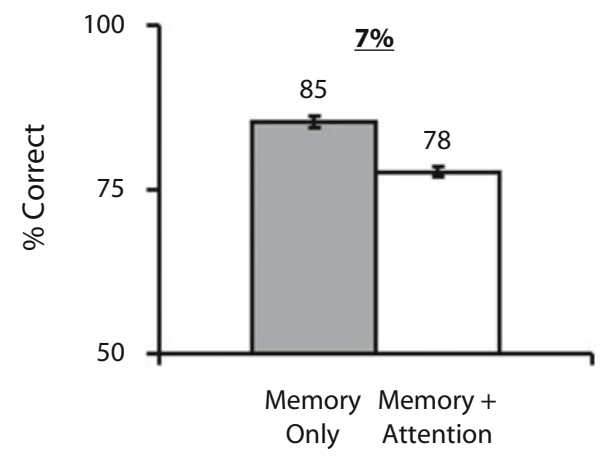

Spatial-Attention/Spatial-Memory

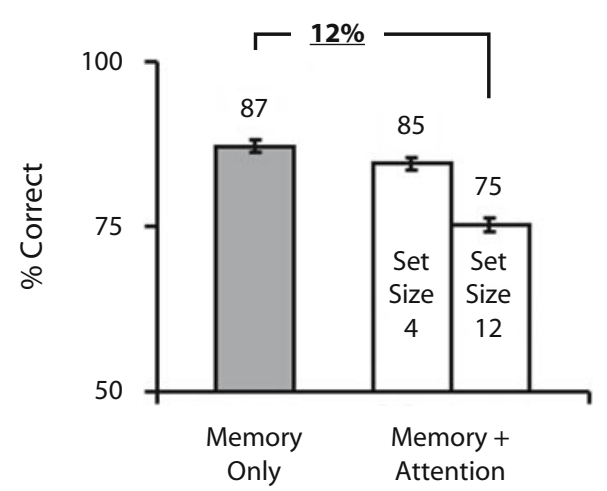

Figure 2. Memory accuracy across all four experiments. Each graph represents the memory-only condition on the left and the memory-and-attention condition on the right. Error bars represent $95 \%$ withinsubjects confidence intervals (Loftus \& Masson, 1994). Underlined percentages represent the size of dualtask interference (accuracy of memory-only trials minus accuracy of memory-and-attention trials). 
Table 1

Mean Accuracy for the Object Feature Report Task and Mean Reaction Times for the Visual Search Task

\begin{tabular}{|c|c|c|c|c|c|c|c|}
\hline \multirow[b]{3}{*}{ Task } & \multirow[b]{3}{*}{ Response } & \multicolumn{6}{|c|}{ Accuracy $(\%)$} \\
\hline & & \multicolumn{3}{|c|}{ Single Task } & \multicolumn{3}{|c|}{ Dual Task } \\
\hline & & $\begin{array}{c}\text { Same } \\
\text { Object }\end{array}$ & $\begin{array}{c}\text { Different } \\
\text { Object }\end{array}$ & Effect & $\begin{array}{l}\text { Same } \\
\text { Object }\end{array}$ & $\begin{array}{c}\text { Different } \\
\text { Object }\end{array}$ & Effect \\
\hline \multirow[t]{2}{*}{ Object-attention/object-memory } & First & 94 & 90 & $4( \pm 0.9)$ & 84 & 81 & $3( \pm 1.3)$ \\
\hline & Second & 93 & 88 & $5( \pm 0.8)$ & 86 & 80 & $6( \pm 1.3)$ \\
\hline \multirow[t]{6}{*}{ Object-attention/spatial-memory } & First & 95 & 93 & $2( \pm 0.7)$ & 90 & 88 & $2( \pm 1.0)$ \\
\hline & Second & 94 & 91 & $3( \pm 0.9)$ & 89 & 85 & $4( \pm 0.9)$ \\
\hline & & \multicolumn{6}{|c|}{ Response Time (msec) } \\
\hline & & \multicolumn{3}{|c|}{ Single Task } & \multicolumn{3}{|c|}{ Dual Task } \\
\hline & & Set & Set & & Set & Set & \\
\hline & Target & Size 4 & Size 12 & Effect & Size 4 & Size 12 & Effect \\
\hline \multirow[t]{2}{*}{ Spatial-attention/object-memory } & Present & 1,032 & 1,247 & $49( \pm 3)$ & 1,122 & 1,517 & $49( \pm 4)$ \\
\hline & Absent & 1,412 & 2,626 & $162( \pm 11)$ & 1,522 & 2,706 & $138( \pm 9)$ \\
\hline \multirow[t]{2}{*}{ Spatial-attention/spatial-memory } & Present & 1,059 & 1,486 & $53( \pm 4)$ & 1,185 & 1,613 & $53( \pm 4)$ \\
\hline & Absent & 1,497 & 2,685 & $154( \pm 8)$ & 1,537 & 2,769 & $149( \pm 9)$ \\
\hline
\end{tabular}

Note-Values within parentheses represent $95 \%$ within-subjects confidence intervals.

fect observed in the spatial-attention/object-memory experiment $[t(94)=5.1, p<.0001]$. By contrast, the $7 \%$ interference effect observed in the object-attention/spatialmemory experiment was significantly smaller than the $12 \%$ interference effect observed in the spatial-attention/ spatial-memory experiment $[t(94)=3.3, p<.001]$.

\section{Attention Task Performance}

To ensure that there were no trade-offs between the memory and attention tasks, we examined performance in the object feature report and visual search tasks; these data appear in Table 1.

\section{Object Feature Report}

In all conditions, observers reported two features that were part of a single object more accurately than two features that were part of two different objects $(p s<.0001)$. However, even though observed object-based effects were larger in the memory-and-attention condition than in the attention-only condition, these differences did not reach significance $[F(1,94)=1.4, p>.05]$.

\section{Visual Search}

Accuracy was above $97 \%$ across all conditions. Reaction times (RTs) increased as set size increased in both the attention-only and the memory-and-attention conditions $(p \mathrm{~s}<.0001)$. The slope was steeper in the targetabsent condition than in the target-present condition $(p<$ $.0001)$. However, the effects of set size (12 or 4$)$ and target type (present or absent) did not differ between task types (attention only or memory and attention) or between experiments (spatial-attention/object-memory or spatialattention/spatial-memory) $(p \mathrm{~s}>.05)$.

One potential methodological concern with our experiments is the possibility that observers might have verbally coded visual stimuli during either the object memory or the object attention task. Although we used a concurrent articulatory suppression task to prevent this strategy, the features and colors used in our experiments might have been verbally recoded, and the results could have been caused by verbal, not visual, interference. To rule out this possibility, we asked observers to remember hard-toverbalize Attneave figures (Attneave \& Arnoult, 1956; see also Barnes et al., 2001), instead of nameable colors. Because the goal of this experiment was to replicate the interference pattern observed in the two conditions that included the color change-detection task, we conducted the object-attention/object-memory experiment and the spatial-attention/object-memory experiment with a within-subjects design (64 trials each) of memory task type (memory only vs. memory and attention). Sixteen observers performed the shape-matching memory task in place of the color change-detection task. As in all the experiments reported above, each trial began with an observer's performing an articulatory suppression task. A six-sided shape, whose size was approximately equal to a colored square, was presented on either side of fixation for $500 \mathrm{msec}$. The position in which a shape appeared was identical to that where a colored square appeared in the experiment reported above. A shape was randomly selected from a list of eight shapes constructed on the basis of Attneave and Arnoult. The shape appeared to the left and right of fixation equally. The test shape always appeared at the location in which it appeared during the memory array presentation. Observers pressed " 1 " if the shape on the memory and test arrays was identical and pressed "2" if these shapes differed.

The shape memory experiment replicated the pattern observed in the color memory experiment (see Figure 3). The accuracy drop from the memory-only condition to the memory-and-attention condition was significant for both experiments $(p<.0001)$. The size of interference was significantly larger when an object memory (shapematching) task was performed with a concurrent object attention task than when it was performed with a spatial attention task $[F(1,15)=8.8, p<.009]$. Observ- 


\section{Object-Attention/Object-Memory}

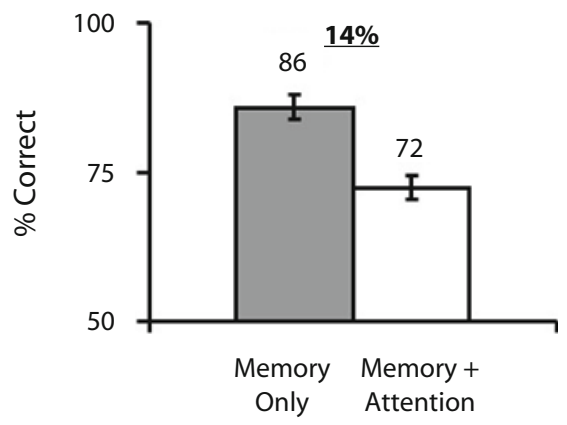

Spatial-Attention/Object-Memory

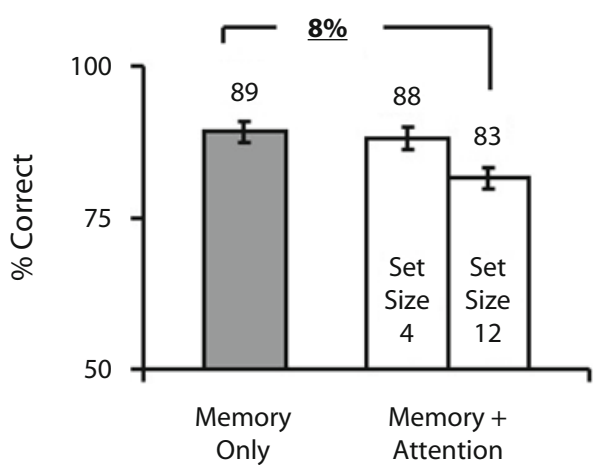

Figure 3. Memory accuracy across the object-attention/objectmemory and spatial-attention/object-memory experiments with shape stimuli.

ers' performance in the object feature report and visual search tasks when the shape memory experiment was concurrently performed is presented in Table $2 .{ }^{3}$ Overall accuracy for visual search was $98 \%$. Because there was no significant difference in observers' dual-task performance between the color memory task and the shape memory task, it is sensible for us to consider that observers probably could not afford to semantically code visual stimuli in our experiments.

\section{DISCUSSION}

We investigated the effect of attention tasks on concurrently performed visual memory tasks. Replicating previous findings (Oh \& Kim, 2004; Woodman \& Luck, 2004), observers' performance of the spatial memory task was interfered with by the visual search task. More importantly, observers' performance of the object memory task was largely interfered with by the object feature report task. The latter results suggest that object-based attention and object-based memory processes may share common selection mechanisms. We propose that some forms of object-based attention arise within object-based visual memory. This proposal is consistent with our earlier suggestion that some object-based selection of attention could operate within late, spatially invariant representations (Vecera, 1997; Vecera \& Farah, 1994).

Importantly, we would not expect an object memory task to be impaired by all kinds of object-based attention tasks. Many object-based effects of attention can be explained by selection from an early object representation in which locations are perceptually grouped together (i.e., grouped array selection; Vecera, 1994). In some cases, selection from a grouped array is perceptually mediated and may not require VSTM representations. Other results from our laboratory support this claim: Lee and Vecera (2005) found that maintenance of four objects in VSTM did not eliminate object-based effects of attention involved in a task that taps into grouped array selection mechanisms (Egly, Driver, \& Rafal, 1994; Vecera, 1994). On the basis of the present results, we predict that the widely used spatially cued object attention task (e.g., Egly et al., 1994) would not cause a substantial decline in object memory accuracy in a dual-task situation similar to the one that we have used here.

We should again acknowledge previous work that attempted to draw conclusions about the locus of objectbased selection. As discussed earlier, the identity of the memory interference observed in Barnes et al. (2001) remains unclear. Because it was not necessary for observers to use VSTM for the primary attention task (vertex judgment), the observed interference might have

Table 2

Mean Accuracy for the Object Feature Report Task and Mean Reaction

Times for the Visual Search Task When the Shape-Matching Task Was Performed in Place of the Color Change-Detection Task

\begin{tabular}{|c|c|c|c|c|}
\hline \multirow[b]{3}{*}{ Task } & \multirow[b]{3}{*}{ Response } & \multicolumn{3}{|c|}{ Accuracy (\%) } \\
\hline & & \multicolumn{2}{|c|}{ Object } & \multirow[b]{2}{*}{ Effect } \\
\hline & & Same & Different & \\
\hline \multirow[t]{5}{*}{ Object-attention/object-memory } & First & 73 & 75 & $2( \pm 4.7)$ \\
\hline & Second & 77 & 69 & $8( \pm 4.3)$ \\
\hline & & \multicolumn{3}{|c|}{ Response Time (msec) } \\
\hline & & \multicolumn{2}{|c|}{ Set Size } & \\
\hline & Target & 4 & 12 & $(\mathrm{msec} / \mathrm{item})$ \\
\hline \multirow[t]{2}{*}{ Spatial-attention/object-memory } & Present & 1,323 & 1,871 & $68( \pm 3)$ \\
\hline & Absent & 1,719 & 2,944 & $153( \pm 7)$ \\
\hline
\end{tabular}

Note-Values within parentheses represent $95 \%$ within-subjects confidence intervals. 
been caused by the interaction between object memory (a shape-matching task) and perceptual-level processes, such as observers' sensitivity to lower level perceptual cues (e.g., convexity). These possibilities suggest that different tasks might produce different levels of interference when an attention task is performed with a concurrent memory task. As mentioned above, we predict that a task measuring object-based attention that operates within observers' perceptual representations (i.e., grouped array selection) should not interfere with a task that measures late, spatially invariant selection (as in the present experiments).

We would also like to note that the dual-task approach we used for the present experiments does not completely dissociate an object's identity and location. However, to determine whether spatially invariant object-based attention operates within VSTM, it is not necessary to perfectly dissociate features that objects possess and locations that objects occupy. Objects cannot exist without occupying any locations; however, how attention selects features that are part of an object and how attention selects a group of locations that objects occupy differ in relative terms. Previous studies have made this point. The feature report task that we used does not require observers to rely on coding or attending to an object's spatial position (Awh et al., 2001; Vecera \& Farah, 1994). The feature report task encourages observers to focus on the featural properties that objects possess, whereas the visual search task facilitates observers to look for a specific location that a target object occupies. In summary, the type of attention observed (object based or location based) might depend on the task that observers perform. The same logic applies to the object memory and spatial memory tasks that we used in the present experiments. Attending to objects with relatively complex features in a display (as in the object feature report task) might permit a late selection from spatially invariant representations to operate, whereas attending to objects with relatively simple features in a cluttered display might facilitate an earlier selection from locationbased (grouped array, if necessary) representations.

The present results have implications for both the nature of late object-based selection and the nature of attention effects during VSTM maintenance. Recent studies have reported that attention selects objects already stored in VSTM (e.g., Griffin \& Nobre, 2003). Specifically, when the number of to-be-remembered items is near or above VSTM capacity, attention seems to protect a cued item from decay in VSTM or interference from other items held in VSTM (Matsukura, Luck, \& Vecera, 2007). The present findings raise the possibility that this selection process from VSTM is object based; attention may protect stored items as integrated object representations rather than as independent feature representations.

\section{AUTHOR NOTE}

This research was made possible by a Sigma Xi Grants-in-Aid of Research Award to M.M. and by National Science Foundation Award 03-39171 to S.P.V. We thank Steve Luck, Andrew Hollingworth, Steve Franconeri, Kyle Cave, Patricia Reuter-Lorenz, and an anonymous re- viewer for their helpful comments, as well as Emily Muff, Shaunna Kulp, Kristine Nurse, Jean Marie Hartin, and Jessica M. Johnson for assistance with data collection. Correspondence concerning this article should be addressed to M. Matsukura, Department of Psychology, University of Iowa, E11 Seashore Hall, Iowa City, IA 52242-1407 (e-mail: michi -matsukura@uiowa.edu.

\section{REFERENCES}

Alvarez, G. A., \& Cavanagh, P. (2004). The capacity of visual shortterm memory is set both by information load and by number of objects. Psychological Science, 15, 106-111.

Attneave, F., \& Arnoult, M. D. (1956). The quantitative study of shape and pattern perception. Psychological Bulletin, 53, 452-471.

Awh, E., Barton, B., \& Vogel, E. K. (2007). Visual working memory represents a fixed number of items regardless of complexity. Psychological Science, 18, 622-628.

Awh, E., Dhaliwal, H., Christensen, S., \& Matsukura, M. (2001). Evidence for two components of object-based selection. Psychological Science, 12, 329-334.

Awh, E., Jonides, J., \& Reuter-Lorenz, P. A. (1998). Rehearsal in spatial working memory. Journal of Experimental Psychology: Human Perception \& Performance, 24, 780-790.

Barnes, L. L., Nelson, J. K., \& Reuter-Lorenz, P. A. (2001). Object-based attention and object-based working memory: Overlapping processes revealed by selective interference effects in humans. In C. Casanova \& M. Ptito (Eds.), Progress in brain research (Vol. 134, pp. 471-481). Amsterdam: Elsevier.

BAYLIS, G. C., \& DRIVER, J. (1993). Visual attention and objects: Evidence for hierarchical coding of location. Journal of Experimental Psychology: Human Perception \& Performance, 19, 451-470.

Besner, D., Davies, J., \& Daniels, S. (1981). Reading for meaning: The effects of concurrent articulation. Quarterly Journal of Experimental Psychology, 33A, 415-437.

Duncan, J. (1984). Selective attention and the organization of visual information. Journal of Experimental Psychology: General, 113, 501517.

EgLy, R., Driver, J., \& Rafal, R. D. (1994). Shifting visual attention between objects and locations: Evidence from normal and parietal lesion subjects. Journal of Experimental Psychology: General, 123, 161-177.

Gajewski, D. A., \& Brockmole, J. R. (2006). Feature bindings endure without attention: Evidence from an explicit recall task. Psychonomic Bulletin \& Review, 13, 581-587.

Gibson, B. S. (1994). Visual attention and objects: One versus two or convex versus concave? Journal of Experimental Psychology: Human Perception \& Performance, 20, 203-207.

Griffin, I. C., \& Nobre, A. C. (2003). Orienting attention to locations in internal representations. Journal of Cognitive Neuroscience, 15, 1176-1194.

Hollingworth, A., Richard, A. M., \& LucK, S. J. (2008). Understanding the function of visual short-term memory: Transsaccadic memory, object correspondence, and gaze correction. Journal of Experimental Psychology: General, 137, 163-181.

IRWIN, D. E. (1992). Memory for position and identity across eye movements. Journal of Experimental Psychology: Learning, Memory, \& Cognition, 18, 307-317.

IRWIN, D. E., \& ANDREWS, R. V. (1996). Integration and accumulation of information across saccadic eye movements. In T. Inui \& J. L. McClelland (Eds.), Attention and performance XVI: Information integration in perception and communication (pp. 125-155). Cambridge, MA: MIT Press, Bradford Books.

Lee, H., \& Vecera, S. P. (2005). Visual cognition influences early vision: The role of visual short-term memory in amodal completion. Psychological Science, 16, 763-768.

Loftus, G. R., \& Masson, M. E. J. (1994). Using confidence intervals in within-subject designs. Psychonomic Bulletin \& Review, 1, 476-490.

LUCK, S. J., \& Vogel, E. K. (1997). The capacity of visual working memory for features and conjunctions. Nature, 390, 279-281.

Macmillan, N. A., \& Creelman, C. D. (2005). Detection theory: A user's guide (2nd ed.). Mahwah, NJ: Erlbaum. 
Matsukura, M., LucK, S. J., \& Vecera, S. P. (2007). Attention effects during visual short-term memory maintenance: Protection or prioritization? Perception \& Psychophysics, 69, 1422-1434.

OH, S.-H., \& KIM, M.-S. (2004). The role of spatial working memory in visual search efficiency. Psychonomic Bulletin \& Review, 11, 275-281.

VECERA, S. P. (1994). Grouped locations and object-based attention: Comment on Egly, Driver, and Rafal (1994). Journal of Experimental Psychology: General, 123, 316-320.

VECERA, S. P. (1997). Grouped arrays versus object-based representations: Reply to Kramer et al. (1997). Journal of Experimental Psychology: General, 126, 14-18.

VeCERA, S. P., \& FARAH, M. J. (1994). Does visual attention select objects or locations? Journal of Experimental Psychology: General, 123, $146-160$.

Vogel, E. K., Woodman, G. F., \& Luck, S. J. (2001). Storage of features, conjunctions, and objects in visual working memory. Journal of Experimental Psychology: Human Perception \& Performance, 27, 92-114.

Woodman, G. F., \& LUCK, S. J. (2004). Visual search is slowed when visuospatial working memory is occupied. Psychonomic Bulletin \& Review, 11, 269-274.

Woodman, G. F., Vogel, E. K., \& Luck, S. J. (2001). Visual search remains efficient when visual working memory is full. Psychological Science, 12, 219-224.

\section{NOTES}

1. The main results that we report in this article were significant with 16 or fewer participants. We collected 48 observers for each experiment to counterbalance the order of the three blocks.

2. To rule out possible distortions from response bias, all the data in this study were also analyzed with $d^{\prime}$, a measure of sensitivity based on signal detection theory (Macmillan \& Creelman, 2005). The analyses of $d^{\prime}$ yielded the same pattern of results as did the analyses of percent correct.

3. When the visual search data (RT) in the spatial-attention/ object(color)-memory experiment are replaced with the spatialattention/object(shape)-memory experiment, the set size $\times$ target type $\times$ experiment (between-subjects) interaction does not reach significance $(p=.94)$. In fact, none of the statistical comparisons that involve a between-subjects factor of experiment is significant. These results indicate that search efficiency observed for the shape load does not reliably differ from search efficiency observed for the spatial memory load.

(Manuscript received February 12, 2008; revision accepted for publication February 12, 2009.) 